\title{
Cellular architecture and transmitter phenotypes of neurons of the mouse median raphe region
}

\author{
Katalin E. Sos ${ }^{1,2}$ - Márton I. Mayer ${ }^{1}$ Csaba Cserép ${ }^{1}$ Flóra S. Takács ${ }^{1}$. \\ András Szőnyi $^{1,2}$ - Tamás F. Freund ${ }^{1} \cdot$ Gábor Nyiri $^{1}$
}

Received: 14 August 2015/Accepted: 16 March 2016/Published online: 4 April 2016

(c) The Author(s) 2016. This article is published with open access at Springerlink.com

\begin{abstract}
The median raphe region (MRR, which consist of MR and paramedian raphe regions) plays a crucial role in regulating cortical as well as subcortical network activity and behavior, while its malfunctioning may lead to disorders, such as schizophrenia, major depression, or anxiety. Mouse MRR neurons are classically identified on the basis of their serotonin (5-HT), vesicular glutamate transporter type 3 (VGLUT3), and gamma-aminobutyric acid (GABA) contents; however, the exact cellular composition of MRR regarding transmitter phenotypes is still unknown. Using an unbiased stereological method, we found that in the MR, $8.5 \%$ of the neurons were 5-HT, $26 \%$ were VGLUT3, and $12.8 \%$ were 5 -HT and VGLUT3 positive; whereas $37.2 \%$ of the neurons were GABAergic, and $14.4 \%$ were triple negative. In the whole MRR, $2.1 \%$ of the neurons were 5 -HT, $7 \%$ were VGLUT3, and $3.6 \%$ were 5-HT and VGLUT3 positive; whereas $61 \%$ of the neurons were GABAergic. Surprisingly, $25.4 \%$ of the neurons were triple negative and were only positive for the neuronal marker NeuN. PET-1/ ePET-Cre transgenic mouse lines are widely used to specifically manipulate only 5-HT containing neurons. Interestingly, however, using the ePET-Cre transgenic mice, we found that far more VGLUT3 positive cells expressed ePET than 5-HT positive cells, and about $38 \%$ of the ePET cells contained only VGLUT3, while more
\end{abstract}

Gábor Nyiri

nyiri.gabor@koki.mta.hu

1 Laboratory of Cerebral Cortex Research, Institute of Experimental Medicine, Hungarian Academy of Sciences, Budapest 1083, Hungary

2 János Szentágothai Doctoral School of Neurosciences, Semmelweis University, Budapest 1085, Hungary than $30 \%$ of 5-HT cells were ePET negative. These data should facilitate the reinterpretation of PET-1/ePET related data in the literature and the identification of the functional role of a putatively new type of triple-negative neuron in the MRR.

Keywords Median raphe Paramedian raphe .

Stereology · Serotonin · 5-HT · ePET · PET-1 · VGLUT3 · VGAT $\cdot$ Immunohistochemistry

\section{Introduction}

The median raphe region (MRR) plays a fundamental role in regulating cortical network activity, as well as subcortical functions (Bohut 1997; Vertes et al. 1999). It participates in several aspects of fear behavior, its lesion disrupts the acquisition of conditioned fear memory, and it is associated with specific subtypes of anxiety disorders (Avanzi and Brandão 2001; Avanzi et al. 2003; Silva et al. 2004; Borelli et al. 2005; Dos Santos et al. 2005; Ohmura et al. 2014; Peters et al. 2014; Zangrossi and Graeff 2014). Its functional alterations may also lead to disorders, such as schizophrenia or major depression (Aghajanian and Marek 2000; Hensler 2006). However, its precise cellular composition with regard to transmitter phenotypes is still unknown.

The MRR is located in the midline of the brainstem and consists of the MR and the paramedian raphe (PMR) subregions. The MRR used to be known as a serotonergic nucleus; however, several studies have already reported the presence of non-serotonergic neurons (Gras et al. 2002; Jackson et al. 2009) as well. Four cell populations can be distinguished based on 5-HT, glutamate, and gammaaminobutyric acid (GABA) contents: (1) some neurons 
contain only 5-HT (serotonin only, SO cells); (2) others show only vesicular glutamate transporter type 3 expression (VGLUT3 only, GO cells); (3) in the third group, both molecules are detectable (serotonin and VGLUT3, SG cells) (Fremeau et al. 2002; Shutoh et al. 2008); (4) while the fourth group of cells is GABAergic (Stamp and Semba 1995; Serrats et al. 2003; Calizo et al. 2011) and only contains vesicular GABA transporter (VGAT). No studies have investigated the possible occurrence of cells that would express none of these molecules or the ratios of these four cell types in the MRR.

Serotonergic and glutamatergic MRR cells project densely to several forebrain areas (Vertes et al. 1999; Azmitia 1978; Köhler 1982; Aznar et al. 2004; Varga et al. 2009). Serotonergic neurons desynchronize the hippocampal activity and disrupt rhythmic discharge of septal cholinergic and GABAergic neurons (Assaf and Miller 1978; Kinney et al. 1996; Vertes and Kocsis 1997). VGLUT3 containing glutamatergic neurons suppress the hippocampal ripple activity and disrupt memory consolidation (Wang et al. 2015), and both of these cells can innervate more forebrain areas simultaneously (Szőnyi et al. 2014). Although serotonergic and glutamatergic neurons are frequently investigated, it is unknown how many of them possess both transmitters.

Efforts to genetically identify and modify serotonergic cells led to the discovery of the E26 transformationspecific transcription factor PET-1 and its enhancer region ePET (Hendricks et al. 1999). The generation of PET-1Cre and ePET-Cre transgenic mouse lines promised efficient manipulation of the 5-HT containing neurons and provided tools for numerous studies (Braz et al. 2009; Hodges et al. 2009; Hawthorne et al. 2010; Liu et al. 2010; Depuy et al. 2011, 2013; Spaethling et al. 2014; Wang et al. 2015).

In this study, we investigated the number of cells of different cell populations in the MRR. In the whole MRR, $2.1 \%$ of the neurons were 5-HT, $7 \%$ were VGLUT3, and $3.6 \%$ were 5 -HT and VGLUT3 positive; whereas $61 \%$ of the neurons were GABAergic. Surprisingly, $25.4 \%$ of the MRR neurons were only positive for the neuronal marker NeuN and were negative for 5-HT, VGLUT3, and VGAT, and because of several reasons detailed below, these cells are highly unlikely to be false negative. Furthermore, using one specific commonly used ePET-Cre transgenic mouse line (Jackson Laboratories), we show that more than half of the SO neurons are negative for ePET, and about $38 \%$ of the ePET cells only contain VGLUT3. Therefore, ePET is not specific to serotonergic cells, since it is present in a portion of SO, GO, SG cells, and also in some of the unidentified neuronal population.

\section{Materials and methods}

\section{Animals and perfusions}

All experiments were performed in accordance with the Institutional Ethical Codex and the Hungarian Act of Animal Care and Experimentation guidelines, which are in concert with the European Communities Council Directive of September 22, 2010 (2010/63/EU). The Animal Care and Experimentation Committee of the Institute of Experimental Medicine of the Hungarian Academy of Sciences and the Animal Health and Food Control Station, Budapest, have also approved the experiments.

VGAT-IRES-Cre mice were crossed with Gt(ROSA)26Sor_CAG/ZsGreen1 or with Gt(ROSA)26Sor_CAG/ tdTomato reporter mice, and ePET-IRES-Cre mice were crossed with Gt(ROSA)26Sor_CAG/ZsGreen1 mice (Jackson Laboratories). Their offspring showed genetically coded specific fluorescent labeling in VGAT or ePET expressing neurons. Scott et al. (2005) described in detail the construction of this ePET-Cre mouse line. Three 42 days old male VGAT-ZsGreen1 offspring mice (named GM1; GM2; and GM3), one 42 days old male VGATtdTomato mouse (named GM4), one 42 days old male C57BL/6J wild-type mouse (named WT), and three 49 days old male ePET-ZsGreen1 mice (named PM1; PM2; and PM3) were used. For perfusion, mice were anesthetized with isoflurane, followed by intraperitoneal injection of an anesthetic mixture (containing $8.3 \mathrm{mg} / \mathrm{ml}$ ketamine, $1.7 \mathrm{mg} / \mathrm{ml}$ xylazine-hydrochloride, and $0.8 \mathrm{mg} /$ $\mathrm{ml}$ promethazinium-chloride) to achieve deep anesthesia. Then, mice were transcardially perfused first with PBS $(0.9 \% \mathrm{NaCl}$ in $0.1 \mathrm{M}$ phosphate buffer) solution for $2 \mathrm{~min}$, followed by $4 \%$ paraformaldehyde for $40 \mathrm{~min}$, and finally with $0.1 \mathrm{M}$ phosphate buffer (PB) for $10 \mathrm{~min}$.

\section{Fluorescent immunohistochemistry}

After perfusion of mice and removal of their brains from the skull, coronal sections were cut on a Leica VT1200S vibratome at $60 \mu \mathrm{m}$ from the whole MRR and collected in stereological order into eight vials. This was followed by washing out the fixative in $0.1 \mathrm{M}(\mathrm{PB})$ for $1 \mathrm{~h}$. Then, the sections were cryoprotected sequentially in $10 \%$ (overnight) and $30 \%(2 \mathrm{~h})$ sucrose in PB and freeze-thawed five times over liquid nitrogen. This was followed by several washing in $0.1 \mathrm{M} \mathrm{PB}(3 \times 10 \mathrm{~min})$ and tris-buffered saline solution (TBS, $3 \times 10 \mathrm{~min}$ ). Then, sections were incubated in TBS containing $1 \%$ human serum albumin, $0.1 \%$ Triton $^{\mathrm{TM}} \mathrm{X}-100$, and $700 \mu \mathrm{g} / \mathrm{ml}$ Digitonin (all from Sigma-Aldrich) for $1 \mathrm{~h}$ to achieve better penetration. 
Vials were separated into two groups (even and odd sections) that were used for experiment type A and B, respectively (Table 2). Even-numbered sections were used to demonstrate the ratios between the 5-HT and/or VGLUT3 positive neurons, while odd-numbered sections were used for determining the number of other neurons.

\section{Antibodies}

Table 1 summarizes information about the primary and secondary antibodies. Previously, we extensively tested the rabbit anti-VGLUT3 antibody in experiments using VGLUT3 knock out animals (Szőnyi et al. 2014). Immunostaining of VGLUT3 in raphe neurons was also tested by others (Mintz and Scott 2006; Shutoh et al. 2008). The mouse anti-NeuN antibody labels a neuron specific DNA binding nuclear protein and is widely used to identify neurons (Mullen et al. 1992). To increase the accuracy of the measurements, we counted only the DAPI stained nuclei of cells. The rat and rabbit anti-5-HT antibodies were characterized before (Amilhon et al. 2010; Fox and Deneris 2012), they labeled the same cells, and also labeled the expected population of neurons, which further confirm their specificity. In the experiment, where the expression pattern of ePET was analyzed, we found a mismatch between ePET and 5-HT expression. These surprising results prompted us to test whether it is possible that some cells only express the synthesizing enzyme of serotonin, but the enzyme does not produce detectable levels of serotonin. Therefore, we tested whether all cells that are positive for the 5-HT synthesizing enzyme, tryptophan hydroxylase (TPH), are also positive for 5-HT. We colocalized rabbit anti-5-HT and mouse anti-TPH labeling (Fig. 2). We found that all 160 examined TPH labeled cells were also positive for 5-HT; consequently, 5-HT was always detected in TPH expressing cells. This shows that the sensitivity of 5-HT labeling cannot be responsible for the lack of labeling in some cells, because cells that express its synthesizing enzyme, TPH, always express detectable levels of 5-HT as well.

The antibody penetration into $60 \mu \mathrm{m}$-thick sections was examined rigorously using confocal imaging, and was found to be perfect even in the middle of the section. Secondary antibodies were extensively tested for possible cross-reactivity with other primary or secondary antibodies, but no cross-reactivity was found.

\section{Confocal microscopy}

Image stacks were recorded by using a Nikon A1R confocal laser-scanning system built on a Ti-E inverted microscope with 0.45 NA CFI Super Plan Fluor ELWD 20XC Nikon objective and operated by NIS-Elements AR 4.3 software. Argon ion laser (457-514 nm, $40 \mathrm{~mW}$ ), yellow DPSS laser $(561 \mathrm{~nm}, 20 \mathrm{~mW})$, violet diode laser $(405 \mathrm{~nm})$, and diode laser system $(647 \mathrm{~nm}, 100 \mathrm{~mW})$ were used as excitation lasers with appropriate filters. Images were acquired at a $\mathrm{z}$-separation of $1 \mu \mathrm{m}$. Each section plane was identified by using the Mouse Brain Atlas (Paxinos and Franklin 2012).

Table 1 Antibody specifications

\begin{tabular}{|c|c|c|c|c|c|c|c|c|c|c|c|}
\hline Raised against & Raised in & \multicolumn{2}{|c|}{$\begin{array}{l}\text { Protein } \mathrm{cc} \text {. of } \\
\text { stock solution }\end{array}$} & \multicolumn{2}{|c|}{ Dilution } & \multicolumn{2}{|c|}{ Source } & Catalog number & Lot number & \multicolumn{2}{|c|}{ Characterized } \\
\hline VGLUT3 & Rabbit & \multicolumn{2}{|c|}{$1 \mu \mathrm{g} / \mu \mathrm{l}$} & \multicolumn{2}{|c|}{$1: 500$} & \multicolumn{2}{|c|}{ Synaptic Systems } & 135203 & & \multicolumn{2}{|c|}{ Szonyi et al. (2014) } \\
\hline $\mathrm{NeuN}$ & Mouse & & & \multicolumn{2}{|c|}{$1: 500$} & \multicolumn{2}{|c|}{ Chemicon } & MAB 377 & LV 1359479 & \multicolumn{2}{|c|}{ Mullen et al. (1992) } \\
\hline Serotonin & Rat & \multicolumn{2}{|c|}{ Not available } & $1: 5$ & & Merc & Gillipore & MAB 352 & 2168248 & \multicolumn{2}{|c|}{ Amilhon et al. (2010) } \\
\hline Serotonin & Rabbit & \multicolumn{2}{|c|}{$25 \mu \mathrm{g} / \mathrm{ml}$} & \multicolumn{2}{|c|}{$1: 10000$} & \multicolumn{2}{|c|}{ ImmunoStar } & 20080 & 1431001 & \multicolumn{2}{|c|}{ Fox and Deneris (2012) } \\
\hline TPH & Mouse & & & & & Sigm & Idrich & T0678 & & Cal & et al. (2011) \\
\hline Conjugated with & Raised i & & Raised a & & & ation & Source & & Catalog numl & & Molecule \\
\hline Alexa 594 & Donkey & & Mouse & & & & Life tech & nologies & A-21203 & & Full IgG \\
\hline Alexa 647 & Chicken & & Rat & & & & Life tech & nologies & A-21472 & & Full IgG \\
\hline Alexa 647 & Donkey & & Rabbit & & & & $\begin{array}{r}\text { Jackson } \\
\text { researc }\end{array}$ & mmuno- & $711-605-152$ & & Full IgG \\
\hline Cy3 & Donkey & & Rabbit & & & & $\begin{array}{r}\text { Jackson } \\
\text { researc }\end{array}$ & mmuno- & 711-165-152 & & Full IgG \\
\hline Alexa 488 & Donkey & & Mouse & & & & Life tech & nologies & A21206 & & Full IgG \\
\hline Alexa 488 & Donkey & & Rabbit & & & & Life tech & nologies & A 21202 & & Full IgG \\
\hline DAPI & - & & - & & & 0.000 & Sigma-A & drich & D9564 & & - \\
\hline
\end{tabular}




\section{Stereology measurement}

Unbiased design-based stereological measurements were carried out using the optical fractionator method (Sterio 1984; Gundersen 1986; West and Slomianka 1991; Schmitz and Hof 2005), which is based on the principle that one can accurately define the number of cells in the volume of interest by counting them in a predetermined fraction of the given volume (Dorph-Petersen et al. 2001). To get the total cell numbers, the number of counted cells is multiplied by the reciprocal of three different fractions: section, area, and thickness sampling fractions (West and Slomianka 1991). Using systematic random sampling in each experiment, every second section of the MRR was used; therefore, section sampling fraction was 0.5. In mounted sections, cells were counted only within a fraction of a predefined grid area. In the MR, this fraction was $15^{2} / 40^{2} \mu \mathrm{m}$ in experiment type $\mathrm{A}$ and $15^{2} / 80^{2} \mu \mathrm{m}$ in experiment type B. In the PMR, this fraction was $10^{2}$ / $80^{2} \mu \mathrm{m}$ for both types of experiments. Finally, thickness sampling fraction was about $15 / 28 \mu \mathrm{m}$, because the average mounted section thickness was about $28 \mu \mathrm{m}$ and counting performed only in a $15-\mu \mathrm{m}$-high counting cube. We used a guard zone of minimum $5 \mu \mathrm{m}$ of tissue above and below the counting cube; however, for maximum accuracy, thickness sampling fractions were determined at every sampling site. Cells were counted inside the counting cubes or if they touched one of the inclusion planes of the counting cubes. Using these parameters, we directly identified the phenotype of about $13 \%$ of the MR neurons and altogether counted about 12,300 nuclei in MRR in these animals. Cell counting was carried out in Stereo Investigator 10.0 stereology software (MBF Bioscience), while cells were identified parallel using NISElements AR 4.2 software.

\section{Results}

\section{Cell types of the MRR}

Using immunohistochemistry combined with stereological methods, we identified ten different types of neuronal phenotypes in the MRR. We used three kinds of genetically modified mouse strains and one wild-type mouse. We carried out two types of experiments, because we could use a maximum of four different fluorescent channels per experiment. In experiment type $\mathrm{A}$, we focused on the identification of $\mathrm{SO}, \mathrm{GO}, \mathrm{SG}, \mathrm{VGAT}$, or ePET positive cells, while in experiment type $\mathrm{B}$, we primarily focused on NeuN positive neurons that were negative for all other labeling (see Table 2). To label 5-HT, VGLUT3, and NeuN, we used immunohistochemistry; to stain the nuclei, we performed DAPI histochemistry and we used genetically expressed fluorescent markers for the visualization of VGAT and ePET. Using an unbiased stereological method, the combination of different mice and two types of experiments allowed the estimation of the absolute number of different cells in the MRR. The general labeling pattern of neuronal markers distributed in the MRR as expected, and neuronal markers could be clearly distinguished (Figs. 1, 2, 3, 4). We found that the genetic background did not have any effect on the estimated cell numbers.

In all mouse strains, genetically determined fluorescent markers showed intensive expression in the soma of VGAT or ePET containing neurons (Figs. 3a3-d3, 4a3, b3). Some neurons expressed only these genetically determined markers: these are only VGAT positive (Fig. 3a3, c3, asterisk) or only ePET positive cells (Fig. 4b3, asterisk). In experiment type $B$, to determine the total number of neurons, we used Neu-N staining (Fig. 3a1, c1), which labeled all neuronal nuclei and with a lower intensity also the cytoplasm. Some

Table 2 Experiment types, primary and secondary antibody combinations, and animals from different mouse strains

\begin{tabular}{|c|c|c|}
\hline Mice (genetic background) & $\begin{array}{l}\text { Experiment type A (here } 5 \text {-HT and VGLUT3 } \\
\text { cells could be distinguished, using different } \\
\text { fluorescent channels) }\end{array}$ & $\begin{array}{l}\text { Experiment type B (here 5-HT and VGLUT3 cells could not } \\
\text { be distinguished, but other neurons could be detected in a } \\
\text { separate channel with NeuN) }\end{array}$ \\
\hline \multirow{2}{*}{$\begin{array}{l}\text { GM1, GM2, GM3 (strain: } \\
\text { VGAT-IRES-Cre-ZsGreen) }\end{array}$} & Rat anti-5-HT/Alexa 647 & Rat anti-5-HT/Alexa 647 \\
\hline & Rabbit anti-VGLUT3/Cy3 & Rabbit anti-VGLUT3/A647 \\
\hline $\begin{array}{l}\text { PM1, PM2, PM3-tested } \\
\text { only with type A (strain: } \\
\text { ePet-ZsGreen) }\end{array}$ & DAPI & $\begin{array}{l}\text { Mouse anti-NeuN/Alexa } 594 \\
\text { DAPI }\end{array}$ \\
\hline \multicolumn{3}{|l|}{$\begin{array}{l}\text { WT-tested only with type } \\
\text { A (strain: } C 57 B L / 6 J)\end{array}$} \\
\hline \multirow{4}{*}{$\begin{array}{l}\text { GM4 (strain: VGAT-IRES- } \\
\text { Cre-tdTomato) }\end{array}$} & Rat anti-5-HT/Alexa 647 & Rat anti-5-HT/Alexa 647 \\
\hline & Rabbit anti-VGLUT3/Alexa 488 & Rabbit anti-VGLUT3/Alexa647 \\
\hline & DAPI & Mouse anti-NeuN/Alexa 488 \\
\hline & & DAPI \\
\hline
\end{tabular}



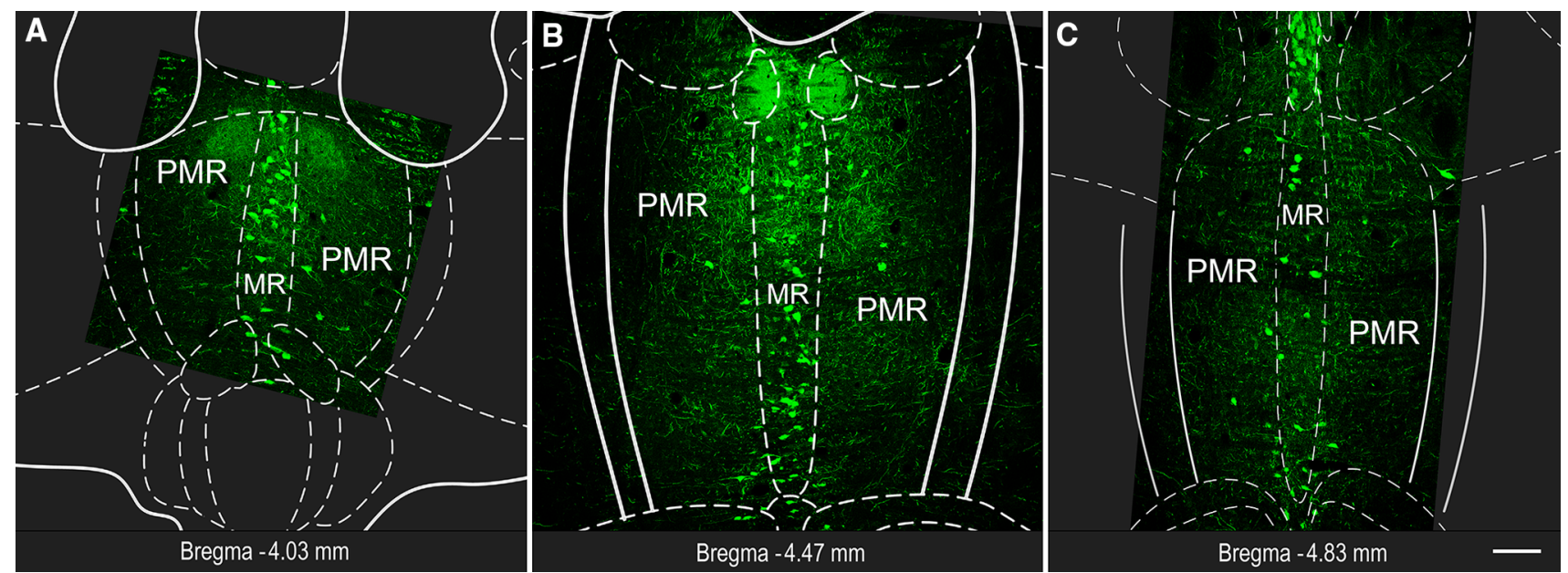

Fig. 1 Fluorescent micrographs show representative MRR sections with 5-HT labeling. Subregions (MR and PMR) are defined based on the Mouse Brain Atlas (Paxinos and Franklin 2012). Position of the coronal section is indicated in each image, relative to the Bregma. Scale bar $100 \mu \mathrm{m}$ for all images
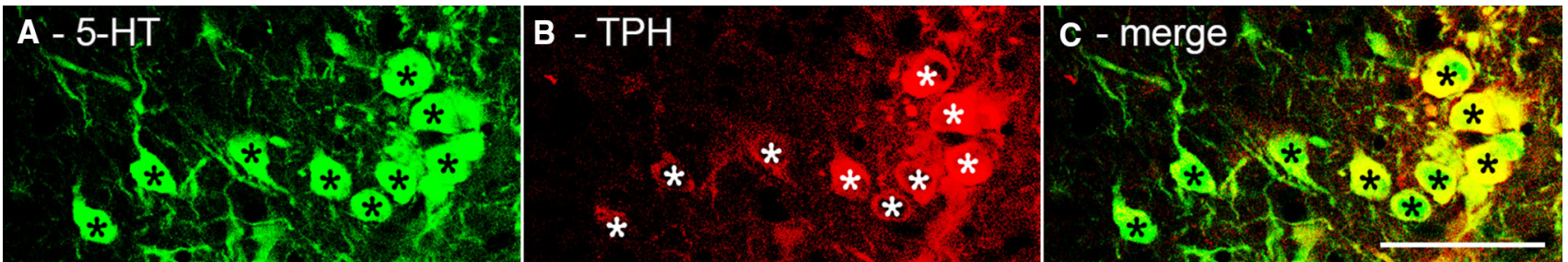

Fig. 2 Confocal laser scanning images (a-c) show $100 \%$ colocalization between the labeling of 5-HT and its synthesizing enzyme, TPH, in representative MR neurons (asterisk). Scale bar $50 \mu \mathrm{m}$ for all images

cells were only NeuN positive, which were marked with filled circle in Fig. 3. In experimental type B, 5-HT and/or VGLUT3 positive cells were visualized in the same fluorescent channel (Fig. 3a2, c2). Some cells were positive for 5-HT and/or VGLUT3 (filled square in Fig. 3), while some were only NeuN positive or VGAT positive.

In experiment type A, 5-HT positive (Figs. 3b1, d1, 4a1, b1) and VGLUT3 positive (Figs. 3b2, d2, 4a2, b2) cells were visualized separately. Both of the markers showed intensive labeling in the somata and axon terminals. In the case of VGAT-Cre animals (Fig. 3), SO cells (filled circle), GO cells (filled square), SG cells (empty circle), VGLUT3 and VGAT positive cells (empty square), and VGATpositive (GABAergic) cells could be differentiated. In the case of ePET-Cre animals (Fig. 4), some SO cells expressed ePET (filled circle), while some were ePET negative (empty circle). Some GO cells were ePET positive (filled square), while some were ePET negative (empty square). Most SG cells were ePET positive (filled diamond), but some were ePET negative (empty diamond). In both types of experiment, we performed DAPI staining, which labelled the nuclei in the blue fluorescent channel.

Stereological results are shown in Table 3 for MR and in Table 4 for PMR and the whole MRR. Based on these data, we calculated the ratios of the different cells types and plotted all results in Fig. 5. Briefly, in the MR, we found that only about $8.5 \%$ of the neurons are SO cells, $26 \%$ are GO cells, and about $12.8 \%$ are SG double positive cells; whereas $37.2 \%$ of the neurons are GABAergic, and $14.4 \%$ of all MR neurons were triple negative for 5-HT, VGLUT3, and VGAT. In the PMR, we found that only $6.4 \%$ of all neurons expressed 5-HT and/or VGLUT3, but these cells appeared only very close to the MR border, which was defined according to the Mouse Brain Atlas (Paxinos and Franklin 2012). $66.8 \%$ of all PMR neurons expressed VGAT, while $27.7 \%$ of the neurons were triple negative for 5-HT, VGLUT3, and VGAT. We never found that colocalization between 5-HT and VGAT and colocalization between VGLUT3 and VGAT occurred only very rarely (see Tables 3, 4; Fig. 5 for details).

The mouse MRR is a relatively small area. Dendritic trees of cells in MR or PMR cross their putative borders as well, making specific activation or deactivation of these putative subregions even more difficult. In addition, separate manipulation of the MR and PMR with optogenetic, electrical, and/or pharmacological tools would currently be a considerable challenge, if possible at all. Although genetic tagging can be used to selectively target a 

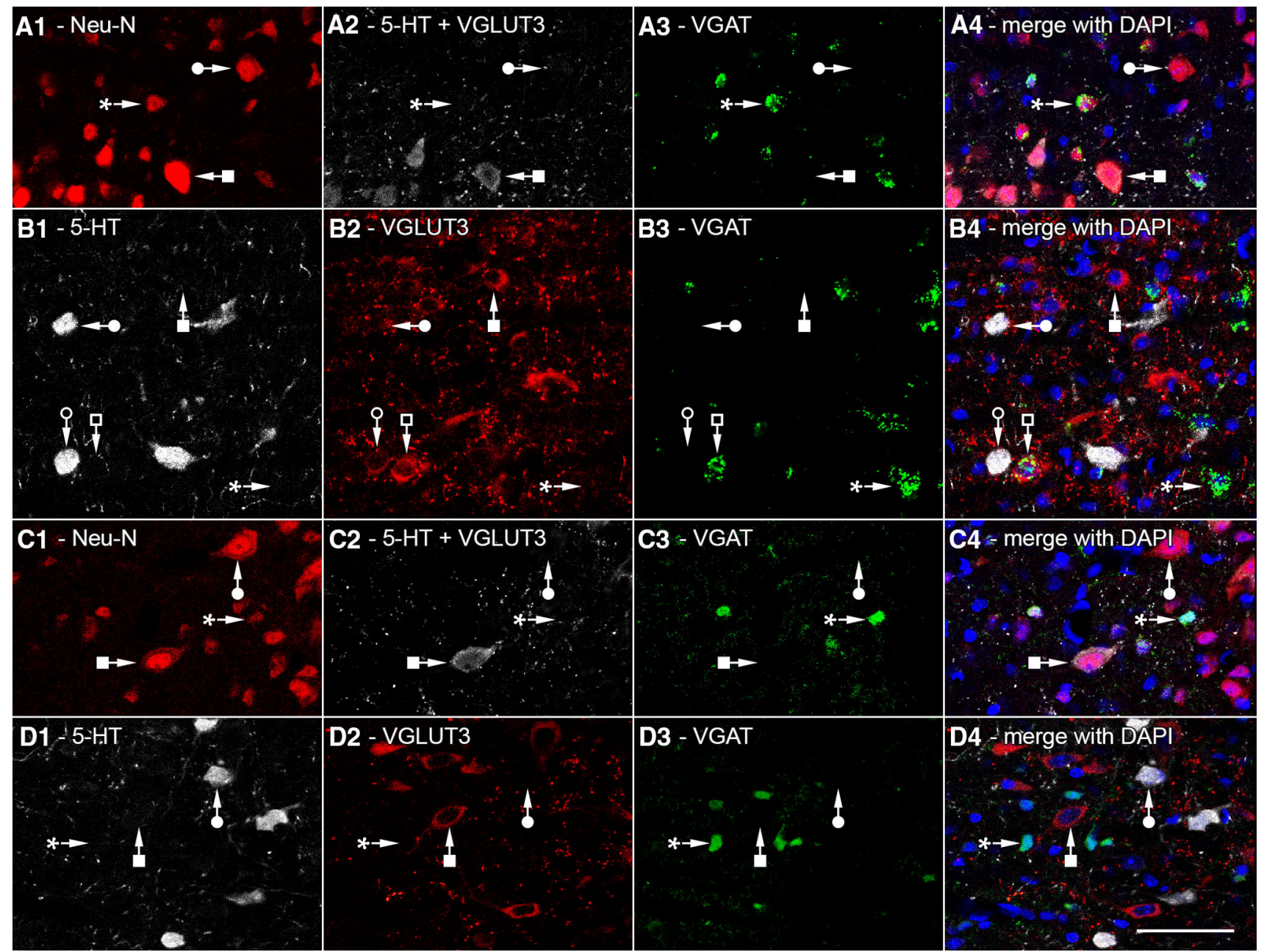

Fig. 3 Confocal laser scanning images used for stereological measurement of different cell types of MRR in VGAT-IRES-Cre-ZsGreen (a, b) and VGAT-IRES-Cre-tdTomato mice (c, d). In both strains, genetically determined fluorescent markers labeling VGAT are shown in green in a3-d3. Nuclei were always labeled with DAPI (blue). In "experiment type B" (a1-a4; c1-c4), neurons were identified with Neu-N staining (red), while 5-HT and/or VGLUT3 positive cells were visualized in the same fluorescent channel (white). Some cells were

subpopulation of neurons, available methods would still manipulate cell in both MR and PMR together. Therefore, separation of MR and PMR is impossible even with optogenetic methods. Therefore, we calculated the number of cells for the whole MRR as well. We found that $13.5 \%$ of the MRR neurons contained 5-HT and/or VGLUT3, while $61.8 \%$ expressed VGAT, and $25.4 \%$ belongs to the unidentified cell type (see Tables 3, 4; Fig. 5 for details).

\section{Distribution of ePET positive cells in the MRR}

Previously, the PET-1 enhancer region, ePET, was thought to be expressed exclusively in serotonergic cells. However, we found a mismatch between ePET and 5-HT expression only NeuN positive (filled circles), and some were positive for 5-HT and/or VGLUT3 (filled square); while some of them only VGAT positive (asterisk). In "experiment type A" (b1-b4; d1-d4), 5-HT positive (white) and VGLUT3 positive (red) cells were visualized separately. SO cells (filled circle), GO cells (filled square), SG cells (empty circle), VGLUT3 and VGAT positive cells (empty square), and only VGAT-positive cells (asterisk) could be differentiated. Scale bar $50 \mu \mathrm{m}$ for all images

in MRR, as shown in Figs. 4, 5 and Tables 3, 4. We also found triple-negative NeuN positive neurons that were labeled with ePET. Although, in this experimental design, colocalization between the genetic markers (ePET and VGAT) is not possible, it is highly unlikely that these markers would colocalize, because ePET positive cells are mostly localized in MR, and most GABAergic cells are in the PMR. In addition, SO and SG cells that are partly ePET positive were never GABAergic. Furthermore, generally, excitatory and inhibitory neurons derive from different cell lines; therefore, it is highly unlikely that ePET would be localized in GABAergic cells as well. Therefore, we classified triple-negative and ePET positive cells as VGAT negative. Results clearly show that ePET is partially 

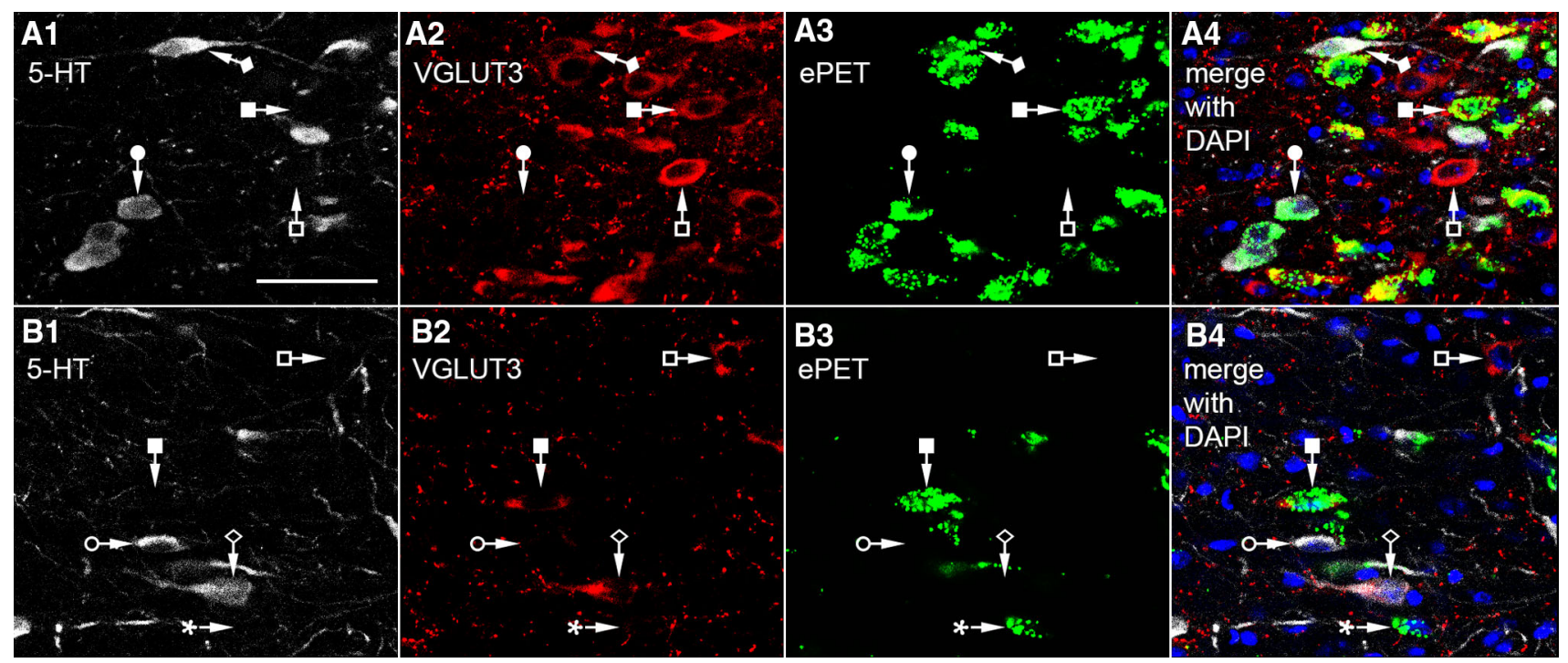

Fig. 4 Confocal laser scanning images used for stereological measurement of different cell types of MRR in ePET-IRES-Cre-ZsGreen mice $(\mathbf{a}, \mathbf{b})$ in "experiment type A". ePET labeling is green, nuclear DAPI labeling is blue, while 5-HT (white) and VGLUT3 (red) positive cells were visualized separately. Some SO cells express ePET (filled circle), while some are ePET negative (empty circle). Some GO

cells were ePET positive (filled square), while some are ePET negative (empty square). Most SG cells are ePET positive (filled diamond), but some are ePET negative (empty diamond). Some cells expressed only ePET without any other labeling (asterisk). Scale bar $50 \mu \mathrm{m}$ for all images

Table 3 Number of cells in median raphe

The number of cells in median raphe in eight mice

\begin{tabular}{|c|c|c|c|c|c|c|c|c|c|}
\hline Labelled cell types & GM1 & GM2 & GM3 & GM4 & WT & PM1 & PM2 & PM3 & Mean \\
\hline Non-neuronal cells, only DAPI positive & 11269 & 10449 & 10672 & 11928 & - & - & - & - & 11079 \\
\hline \multicolumn{9}{|c|}{ All neurons (calculated, using all mice, from the mean number of all labelled neurons) } & 8160 \\
\hline $\begin{array}{l}\text { All 5-HT positive cells, without VGLUT3 labeling-abbreviated as } \\
\text { "SO" }\end{array}$ & 1310 & 515 & 573 & 662 & 640 & 807 & 351 & 697 & 695 \\
\hline And from these, ePET positive cells are: & - & - & - & - & - & 380 & 240 & 378 & 333 \\
\hline $\begin{array}{l}\text { All VGLUT3 positive cells, without 5-HT labeling-abbreviated as } \\
\text { "GO" }\end{array}$ & 1840 & 2078 & 2209 & 2055 & 2041 & 2209 & 2591 & 1957 & 2123 \\
\hline And from these, ePET positive cells are: & - & - & - & - & - & 906 & 886 & 959 & 917 \\
\hline All 5-HT and VGLUT3 double positive cells-abbreviated as "SG" & 627 & 890 & 904 & 940 & 1408 & 1427 & 1014 & 1126 & 1042 \\
\hline And from these, ePET positive cells are: & - & - & - & - & - & 1002 & 705 & 891 & 866 \\
\hline All 5-HT and/or VGLUT3positive cells & 3655 & 3450 & 3426 & 3620 & 4089 & 4862 & 3957 & 3780 & 3855 \\
\hline All VGAT positive cells, without 5-HT and VGLUT3 labeling & 3105 & 3190 & 2619 & 3222 & - & - & - & - & 3034 \\
\hline All VGAT and VGLUT3 double positive cells, without 5-HT labeling & 35 & 149 & 93 & 65 & - & - & - & - & 86 \\
\hline $\begin{array}{l}\text { All NeuN positive cells (triple negative), without 5-HT, VGLUT3 and } \\
\text { VGAT labeling }\end{array}$ & 597 & 712 & 1632 & 1782 & - & - & - & - & 910 \\
\hline All ePET positive cells, without 5-HT and/or VGLUT3 labeling & - & - & - & - & - & 68 & 451 & 296 & 271 \\
\hline
\end{tabular}

expressed in four different populations of neurons (Table 3; Fig. 5).

Because the "border" between MR and PMR is artificial, we have all reason to believe that all SO cells in MRR belong to the same neuronal population, regardless of their localization in MR or PMR, and the same is true for all other cell types. Therefore, the ratio of ePET positive cells in the PMR was calculated based on the ratios found in MR. However, to further confirm that ePET positive cells indeed comprise the same cell types at a similar distribution in the PMR, we defined the phenotype of the counted ePET positive cells in the PMR in experimental type A of one mouse. In that case, we used a semiquantitative approach that allowed the testing of colocalization of other 
Table 4 Number of cells in paramedian raphe and median raphe region

\begin{tabular}{|c|c|c|c|c|c|c|}
\hline \multicolumn{6}{|l|}{ The number of cells in paramedian raphe } & \multirow{2}{*}{$\begin{array}{l}\text { The number of } \\
\text { cells in MRR (sum } \\
\text { of the means of MR } \\
\text { and PMR cells) }\end{array}$} \\
\hline Labelled cell types & GM1 & GM2 & GM3 & GM4 & Mean & \\
\hline Non-neuronal cells, only DAPI positive & 69924 & 59634 & 71573 & 71472 & 68151 & 79230 \\
\hline \multicolumn{5}{|c|}{ All neurons (calculated, using all mice, from the mean number of all labelled neurons) } & 39298 & 47458 \\
\hline All 5-HT positive cells, without VGLUT3 labeling-abbreviated as "SO" & 264 & 0 & 795 & 221 & 320 & 1015 \\
\hline \multicolumn{5}{|c|}{ And from these, ePET positive cells (calculated based on the proportions in Fig. $5 \mathrm{~b}$ ) are: } & 153 & 486 \\
\hline All VGLUT3 positive cells, without 5-HT labeling—abbreviated as "GO" & 751 & 1143 & 2094 & 899 & 1222 & 3344 \\
\hline \multicolumn{5}{|c|}{ And from these, ePET positive cells (calculated based on the proportions in Fig. 5b) are: } & 528 & 1445 \\
\hline All 5-HT and VGLUT3 double positive cells-abbreviated as "SG" & 1438 & 217 & 555 & 460 & 668 & 1710 \\
\hline \multicolumn{5}{|c|}{ And from these, ePET positive cells (calculated based on the proportions in Fig. 5b) are: } & 555 & 1421 \\
\hline All 5-HT and/or VGLUT3positive cells & 2514 & 1535 & 3013 & 1785 & 2212 & 6067 \\
\hline All VGAT positive cells, without 5-HT and VGLUT3 labeling & 33486 & 25728 & 25035 & 19445 & 25924 & 28958 \\
\hline All VGAT and VGLUT3 double positive cells, without 5-HT labeling & 244 & 578 & 234 & 130 & 297 & 383 \\
\hline $\begin{array}{l}\text { All NeuN positive cells(triple-negative cells), without 5-HT, VGLUT3 and } \\
\text { VGAT labeling }\end{array}$ & 9017 & 8646 & 11991 & 13820 & 10710 & 12049 \\
\hline \multicolumn{5}{|c|}{ All ePET positive cells, without 5-HT and/or VGLUT3 labeling (calculated based on the proportions in Fig. 5b) } & 159 & 430 \\
\hline
\end{tabular}

markers with ePET positive cell on a larger number of cells. We collected ePET positive cells in PMR from a representative series of sections (a sample size was 425 ePET+ cells) in experimental type A. This was more optimal in this case, because ePET positive cells are rare in the PMR, and testing with the fully quantitative method would have provided an unacceptably small sample. The result is shown in Fig. $5 d$ lower pie chart. This proportion is very similar to the ratio in the MR (Fig. 5d upper pie chart) that further confirms that these regions contain the same type of cells.

\section{Discussion}

Using an unbiased stereological method, first, we estimated the average numbers of different cell types of the mouse MR and PMR areas. Second, we found that about a quarter of the neurons are negative for 5-HT, VGLUT3, and VGAT, which indicates the existence of a so far unrecognized cell population. Third, we found that ePET is not specific for 5-HT, because it is not present in all SO neurons, and it is expressed in GO and triple-negative neurons as well.

\section{Ratios of cell types in MR and PMR}

MRR is a widely investigated brain area, and several physiological experimental manipulations (excitation, inhibition or lesions) target the whole MRR. The physiological role of individual cell populations could be studied using optogenetic manipulation of cells with certain neurochemical phenotypes; however, even this method cannot be cell type specific, because MRR neurons form neurochemically overlapping cell populations that hamper their separate investigation. Although intersectional genetics might help to separate these cells in the future, it is important to know their ratios; otherwise, even intersectional genetic experiments may be misinterpreted. In addition, our results can help to define the absolute number of cells that are modulated in optogenetic experiments.

5-HT and VGLUT3 neurons are abundant in the center of the MRR, and they are getting gradually less and less abundant outward from the center, and the steepness of the decrease in abundance is variable between mice. Therefore, there is no way to unbiasedly draw a border between MR/ PMR based on staining pattern. Dendritic trees of cells in MR or PMR cross their putative borders as well making specific activation or deactivation of these putative subregions even more difficult, if possible at all. The mouse MRR is relatively small; therefore, even from the practical point of view, the separation of the MR and PMR may also seems to be less useful, because most types of experimental interventions would affect both MR and PMR in mice. These subregions contain the same types of cells, and during experiments using optical fibers the lateral and the conical light spread is about 500-1000 $\mu \mathrm{m}$ from the optical fiber tip (Adamantidis et al. 2007; Yizhar et al. 2011), which would affect the whole volume of median raphe region; therefore, at least in mice, data for the whole MRR seem to be more useful. However, because of historical reasons and also to allow a certain level of comparison with 
A
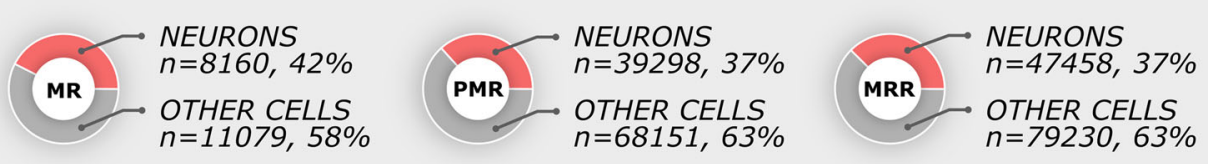

B
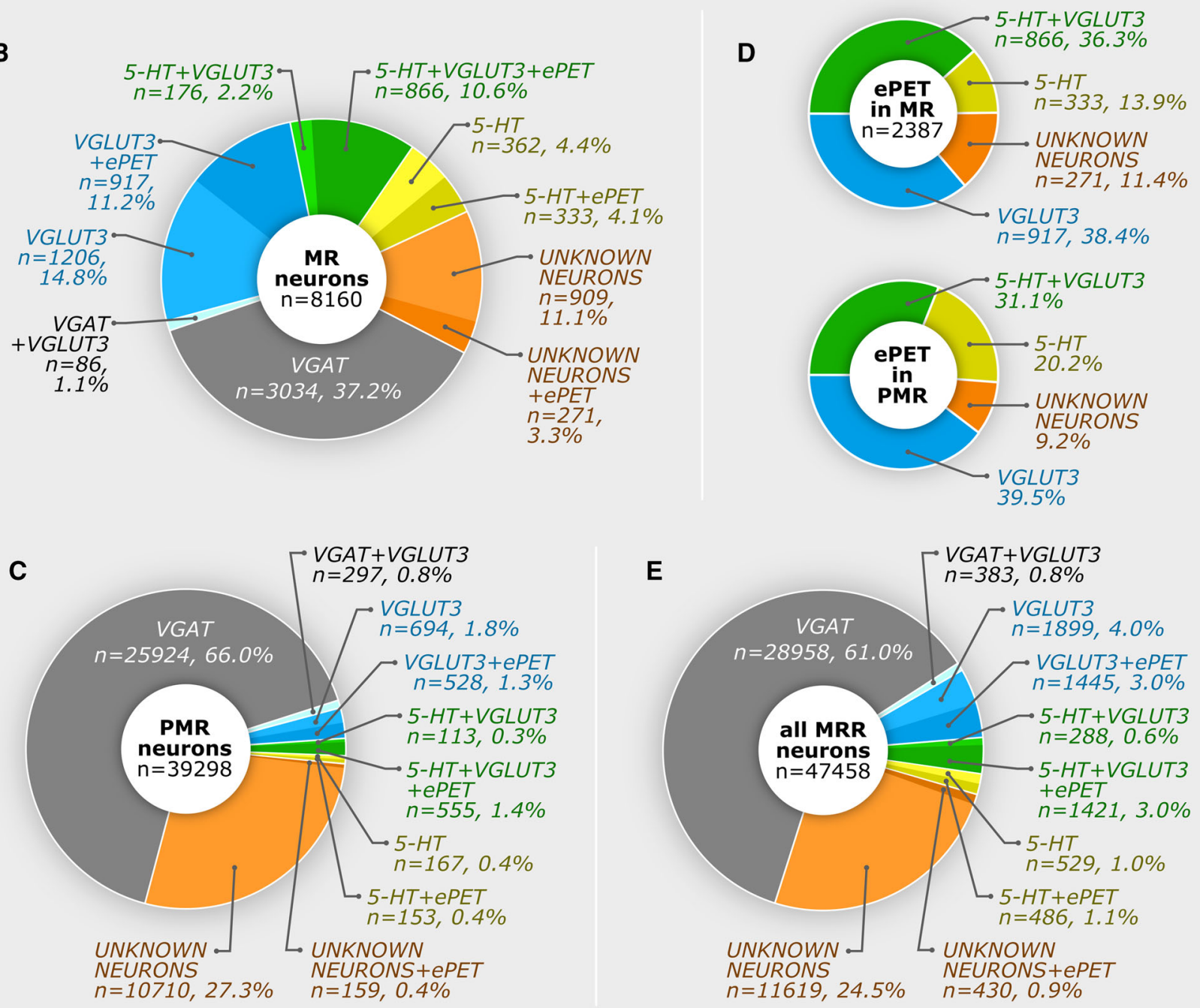

Fig. 5 Number of cells and ratios in the median raphe region. Using original data presented in Tables 3, 4, these pie charts show calculated results originating from eight mice. a Ratios between neurons and non-neuronal cells in the MR, PMR, and MRR. b, c Different neuron types and ePET expression in the MR and PMR,

other species, we analyzed MR and PMR separately as well, for which the only unbiased and reproducible method was to use the stereotaxic Mouse Brain Atlas as reference (Paxinos and Franklin 2012).

Interestingly, based on these criteria, more than a third of the 5-HT and/or VGLUT3 containing neurons are in the PMR - mostly closer to the MR border. We found that about half of the serotonergic cells contained VGLUT3, respectively. d Distribution of 5-HT and VGLUT3 contents in ePET expressing neurons in the MR and PMR. Ratios of PMR neurons were defined with a semiquantitative method; therefore, the number of cells is not shown, but could be calculated from the other pie charts. e Summary pie chart about the whole MRR neurons

whereas a third of the VGLUT3 neurons were positive for 5-HT as well. It is important to note that, while the MRR contains about 47,500 neurons, only about $13 \%$ of them are investigated regularly, because these 5-HT and/or VGLUT3 positive cells are known to project to forebrain areas.

One-third of MR neurons and two-thirds of PMR neurons are GABAergic, and these cells never contained 5-HT. 
Although it may seem insignificant, we found that about $0.8 \%$ of MRR neurons showed VGAT and VGLUT3 double positivity. This type of colocalization is also known in a population of hippocampal GABAergic interneuron (Somogyi et al. 2004), but their connections and role in the MRR is unknown.

\section{The quarter of MRR neurons belong to an unknown population}

We found a previously unidentified cell population, which constituted more than $25 \%$ of the MRR neurons. This group was triple negative for 5-HT, VGLUT3, and VGAT labeling, and was positive only for the neuronal NeuN staining.

These cells are highly unlikely to be false negative, because: (1) they were just as numerous close to the section surface, where no antibody penetration problems occur, (2) they were surrounded by other strongly positive cells, also indicating appropriate antibody penetration; furthermore, (3) these triple-negative cells were distributed homogeneously in the tissue, which means that it is not due to regional penetration differences. In addition, (4) about $28 \%$ of neurons belong to this triple-negative population also in the PMR, where SO, GO, and SG cells are rare, so they are highly unlikely to be false negative of those populations. (5) We proved that 5-HT can indeed label all serotonergic cells, because all TPH positive cells were 5-HT positive. (6) Genetic labeling was always very strong, and the specificity of genetically labeled VGAT expression was extensively tested (Madisen et al. 1993; Vong et al. 2011).

This triple-negative cell type was completely overlooked in the literature, because only SO, GO, SG, and GABAergic neurons were investigated, and to the best of our knowledge, these markers have never been investigated in the same experiment. Triple-negative cells are unlikely to be glutamatergic, because other vesicular glutamate transporter (VGLUT1 or VGLUT2) positive cells have not yet been shown in the MRR (Hioki et al. 2010). Dopaminergic neurons of negligible density were observed in the rat MRR (Jahanshahi et al. 2013), and although its colocalization with 5-HT or VGLUT3 was not tested, triple-negative cells are much more abundant, therefore, highly unlikely to be dopaminergic. In the MRR, most neurokinin-1 receptor labeling did not colocalize with VGLUT3 (Commons and Serock 2009), but other neurons of the MRR were not tested. Somatostatin and galanin positive neurons were also observed in the MRR (Araneda et al. 1999), but their localization in VGLUT3 and/or all 5-HT positive cells was not tested. To the best of our knowledge, so far, no other types of neurons were identified in the MRR that could account for the amount of this new triple-negative type of neuron.

\section{Possible neurotransmitter phenotype plasticity of 5-HT and/or VGLUT3 containing neurons}

Neurotransmitter phenotype plasticity has already been described in several areas of the mammalian brain (Baudry et al. 2010; Dulcis et al. 2013). For instance, during the normal development (between P15 and P90), dopamine neurons lose their VGLUT2 contents completely in ventral tegmental area and substantia nigra (Bérubé-Carrière et al. 2009). In the medial nucleus of the trapezoid body, GABAergic neurons transiently release glutamate, and then switch to glycine as a primary neurotransmitter (Demarque and Spitzer 2012). Glutamatergic granule cells of the dentate gyrus are initially GABAergic, and then express a dual GABAergic/glutamatergic phenotype before becoming purely glutamatergic, but they can transiently express a GABAergic phenotype when a state of hyperexcitability is induced in the adult rat (Gutiérrez et al. 2003). Interestingly, in the MRR in this study, the number of 5-HT and/or VGLUT3 containing neurons shows high variability among mice. Although their ages were similar, 5-HT could be detected in more cells in mouse GM1 compared with GM2; on the other hand, GM2 had more VGLUT3 positive GO and GS cells. However, the total numbers of 5-HT and/or VGLUT3 containing neurons are fairly stable among animals, which suggest that in some mice, serotonergic/glutamatergic ratios would change without changing the total number of main forebrain projection neurons: $\mathrm{SO}, \mathrm{GO}$, and SG cells. These shifts in ratios are unlikely to be due to technical reasons, because we used all sections of the MRR and collected a large systematic random sample; therefore, uneven or topographic cell distributions could not account for this variability. We may hypothesize that these $\mathrm{SO}, \mathrm{GO}$, and SG cells belong to the same neuronal population, which can change its transmitter phenotype, as a function of activity in MRR inputs. Other data also support this hypothesis. First, ePET was expressed in all these three types of neurons, and we found some triple-negative cells that were also labeled with ePET, which may represent either a transitional cell population or a developmental side-branch. Second, VGLUT3 accelerates 5-HT transmission at specific 5-HT terminals; its deletion decreased 5-HT tone in projection areas and decreased serotonin autoreceptor-mediated transmission in raphe, further suggesting a close cooperation of these transmitter systems in the same cells (Amilhon et al. 2010). Third, a robust change in serotonin content of raphe neurons has already been detected between postnatal day 22 and 61 (Rind et al. 2000). Our mice (P42, P49) had slightly different housing 
environments; some of them had many littermates, and others had less that may have caused a variation in regulation of their MRR.

\section{Expression of ePET is not restricted to serotonergic neurons}

A large body of the literature, partly summarized by Deneris (2011), is based on the assumption that PET-1/ ePET can faithfully identify serotonergic neurons. PET-1 was identified as a key factor that triggers the appearance of serotonergic phenotype (Hendricks et al. 1999). PET-1 was thought to play a role in the 5-HT neuron development and is required for anxiety-like and aggressive behavior (Hendricks et al. 2003). Based on those results, ePET (an enhancer region upstream of mouse PET-1-transcribed sequences) was identified and was thought to be a reliable marker for serotonergic cells (Scott et al. 2005).

Using a commonly used ePET-Cre transgenic mouse line that was described by Scott et al. (2005) and is now supplied by Jackson Laboratories; here, we studied the expression of ePET in different types of MRR neurons. Surprisingly, ePET is expressed not only in SO cells, but in GO and SG neurons as well. More than half of SO cells showed a lack of ePET expression, while almost half of GO neurons were also ePET positive. More than a tenth of all ePET expressing neurons were negative for both 5-HT and VGLUT3 labeling. In fact, a previous study has already also shown that 5-HT positive neurons are present in the raphe even in PET-1 knock-out mice, and they were estimated to be about $20-30 \%$ of the 5-HT neuron population in wild-type mice (Kiyasova et al. 2011). PET-1-independent and dependent serotonergic cells have been shown to project to different brain areas (Kiyasova et al. 2011). Furthermore, non-serotonergic neurons were also found to be positive for PET-1 in several raphe nuclei (Pelosi et al. 2014).

Our results can be used to estimate the number and ratios of modulated neurons in previous and future studies, employing ePET/PET-1 transgenic mice, and facilitate the reinterpretations of data in the literature.

Acknowledgments We thank Emőke Szépné Simon, Katalin Lengyel and Katalin Iványi for the excellent technical assistance. The authors wish to thank László Barna, the Nikon Microscopy Center at IEM, Nikon Austria GmbH and Auro-Science Consulting Ltd for kindly providing technical support for fluorescent microscopy. This work was supported by the European Research Council (Grant number ERC-2011-ADG-294313, SERRACO) and the National Institutes of Health (Grant number NS030549). Gábor Nyiri was supported by a János Bolyai Research Scholarship. András Szőnyi was supported by the European Union and the State of Hungary, cofinanced by the European Social Fund in the framework of TÁMOP 4.2.4. A/1-11-1-2012-0001 "National Excellence Program".

\section{Compliance with ethical standards}

Conflict of interest The authors declare that they have no conflict of interest.

Open Access This article is distributed under the terms of the Creative Commons Attribution 4.0 International License (http://crea tivecommons.org/licenses/by/4.0/), which permits unrestricted use, distribution, and reproduction in any medium, provided you give appropriate credit to the original author(s) and the source, provide a link to the Creative Commons license, and indicate if changes were made.

\section{References}

Adamantidis AR, Zhang F, Aravanis AM et al (2007) Neural substrates of awakening probed with optogenetic control of hypocretin neurons. Nature 450:420-424. doi:10.1038/ nature 06310

Aghajanian GK, Marek GJ (2000) Serotonin model of schizophrenia: emerging role of glutamate mechanisms. Brain Res Rev 31:302-312

Amilhon B, Lepicard E, Renoir T et al (2010) VGLUT3 (vesicular glutamate transporter type 3 ) contribution to the regulation of serotonergic transmission and anxiety. J Neurosci 30:2198-2210. doi:10.1523/JNEUROSCI.5196-09.2010

Araneda S, Gysling K, Calas A (1999) Raphe serotonergic neurons projecting to the olfactory bulb contain galanin or somatostatin but not neurotensin. Brain Res Bull 49:209-214. doi:10.1016/ S0361-9230(99)00055-6

Assaf SY, Miller JJ (1978) The role of a raphe serotonin system in the control of septal unit activity and hippocampal desynchronization. Neuroscience 3:539-550. doi:10.1016/03064522(78)90018-0

Avanzi V, Brandão ML (2001) Activation of somatodendritic 5-HT1A autoreceptors in the median raphe nucleus disrupts the contextual conditioning in rats. Behav Brain Res 126:175-184. doi:10.1016/S0166-4328(01)00254-6

Avanzi V, Silva RCB, Macedo CE, Brandão ML (2003) 5-HT mechanisms of median raphe nucleus in the conditioned freezing caused by light/foot-shock association. Physiol Behav 78:471-477. doi:10.1016/S0031-9384(03)00029-5

Azmitia CSM (1978) An Autoradiographic analysis of the differential ascending projections of the dorsal and median raphe nuclei in the rat. J Comp Neurol 3:641-667

Aznar S, Qian ZX, Knudsen GM (2004) Non-serotonergic dorsal and median raphe projection onto parvalbumin- and calbindincontaining neurons in hippocampus and septum. Neuroscience 124:573-581. doi:10.1016/j.neuroscience.2003.12.020

Baudry A, Mouillet-Richard S, Schneider B et al (2010) miR-16 targets the serotonin transporter: a new facet for adaptive responses to antidepressants. Science 329:1537-1541. doi:10. 1126/science. 1193692

Bérubé-Carrière N, Riad M, Dal Bo G et al (2009) The dual dopamine-glutamate phenotype of growing mesencephalic neurons regresses in mature rat brain. J Comp Neurol 517:873-891. doi: $10.1002 /$ cne. 22194

Bohut M-C (1997) Serotonin receptors in cognitive behaviors. Cogn Behav 7:243-254

Borelli KG, Gárgaro AC, Dos Santos JM, Brandão ML (2005) Effects of inactivation of serotonergic neurons of the median raphe nucleus on learning and performance of contextual fear conditioning. Neurosci Lett 387:105-110. doi:10.1016/j.neulet.2005. 07.031 
Braz JM, Enquist LW, Basbaum AI (2009) Inputs to serotonergic neurons revealed by conditional viral transneuronal tracing. J Comp Neurol 514:145-160. doi:10.1002/cne.22003

Calizo LH, Akanwa A, Ma X et al (2011) Raphe serotonin neurons are not homogenous: electrophysiological, morphological and neurochemical evidence. Neuropharmacology 61:524-543. doi:10.1016/j.neuropharm.2011.04.008

Commons KG, Serock MR (2009) Coincidence of neurokinin 1 receptor with the vesicular glutamate transporter 3 (VGLUT3) in the rat forebrain. Neurosci Lett 464:188-192. doi:10.1016/j. neulet.2009.08.042

Demarque M, Spitzer NC (2012) Neurotransmitter phenotype plasticity: an unexpected mechanism in the toolbox of network activity homeostasis. Dev Neurobiol 29:997-1003. doi:10.1016/ j.biotechadv.2011.08.021.Secreted

Deneris ES (2011) Molecular genetics of mouse serotonin neurons across the lifespan. Neuroscience 197:17-27. doi:10.1016/j. neuroscience.2011.08.061

Depuy SD, Kanbar R, Coates MB et al (2011) Control of breathing by raphe obscurus serotonergic neurons in mice. $\mathrm{J}$ Neurosci 31:1981-1990. doi:10.1523/JNEUROSCI.4639-10.2011

DePuy SD, Stornetta RL, Bochorishvili G et al (2013) Glutamatergic neurotransmission between the $\mathrm{C} 1$ neurons and the parasympathetic preganglionic neurons of the dorsal motor nucleus of the vagus. J Neurosci 33:1486-1497. doi:10.1523/JNEUROSCI. 4269-12.2013

Dorph-Petersen KA, Nyengaard JR, Gundersen HJG (2001) Tissue shrinkage and unbiased stereological estimation of particle number and size. J Microsc 204:232-246. doi:10.1046/j.13652818.2001.00958.x

Dos Santos L, De Andrade TGCS, Zangrossi H (2005) Serotonergic neurons in the median raphe nucleus regulate inhibitory avoidance but not escape behavior in the rat elevated T-maze test of anxiety. Psychopharmacology 179:733-741. doi:10.1007/ s00213-004-2120-3

Dulcis D, Jamshidi P, Leutgeb S, Spitzer NC (2013) Neurotransmitter switching in the adult brain regulates behavior. Science 340:449-453. doi:10.1126/science. 1234152

Fox SR, Deneris ES (2012) Engrailed is required in maturing serotonin neurons to regulate the cytoarchitecture and survival of the dorsal raphe nucleus. J Neurosci 32:7832-7842. doi:10.1523/ JNEUROSCI.5829-11.2012

Fremeau RT, Burman J, Qureshi T et al (2002) The identification of vesicular glutamate transporter 3 suggests novel modes of signaling by glutamate. Proc Natl Acad Sci USA 99:14488-14493. doi:10.1073/pnas.222546799

Gras C, Herzog E, Bellenchi GC et al (2002) A third vesicular glutamate transporter expressed by cholinergic and serotoninergic neurons. J Neurosci 22:5442-5451

Gundersen HJG (1986) Stereology of arbitrary shaped particles: unbiased estimation of number and size. Science on form. In: Proceeding of the first international symposium for science on form, pp 509-516

Gutiérrez R, Romo-Parra H, Maqueda J et al (2003) Plasticity of the GABAergic phenotype of the "glutamatergic" granule cells of the rat dentate gyrus. J Neurosci 23:5594-5598

Hawthorne AL, Wylie CJ, Landmesser LT et al (2010) Serotonergic neurons migrate radially through the neuroepithelium by dynamin-mediated somal translocation. J Neurosci 30:420-430. doi:10.1523/JNEUROSCI.2333-09.2010

Hendricks T, Francis N, Fyodorov D, Deneris ES (1999) The ETS domain factor Pet- 1 is an early and precise marker of central serotonin neurons and interacts with a conserved element in serotonergic genes. J Neurosci 19:10348-10356

Hendricks TJ, Fyodorov DV, Wegman LJ et al (2003) Pet-1 ETS gene plays a critical role in 5-HT neuron development and is required for normal anxiety-like and aggressive behavior. Neuron 37:233-247. doi:10.1016/S0896-6273(02)01167-4

Hensler JG (2006) Serotonergic modulation of the limbic system. Neurosci Biobehav Rev 30:203-214. doi:10.1016/j.neubiorev. 2005.06.007

Hioki H, Nakamura H, Ma YF et al (2010) Vesicular glutamate transporter 3-expressing nonserotonergic projection neurons constitute a subregion in the rat midbrain raphe nuclei. J Comp Neurol 518:668-686. doi:10.1002/cne.22237

Hodges MR, Wehner M, Aungst J et al (2009) Transgenic mice lacking serotonin neurons have severe apnea and high mortality during development. J Neurosci 29:10341-10349. doi:10.1523/ JNEUROSCI.1963-09.2009

Jackson J, Bland BH, Antle MC (2009) Nonserotonergic projection neurons in the midbrain raphe nuclei contain the vesicular glutamate transporter VGLUT3. Synapse 63:31-41. doi:10.1002/ syn. 20581

Jahanshahi A, Steinbusch HWM, Temel Y (2013) Distribution of dopaminergic cell bodies in the median raphe nucleus of the rat brain. J Chem Neuroanat 53:60-63. doi:10.1016/j.jchemneu. 2013.09.002

Kinney GG, Kocsis B, Vertes RP (1996) Medial septal unit firing characteristics following injections of $8-\mathrm{OH}-\mathrm{DPAT}$ into the median raphe nucleus. Brain Res 708:116-122

Kiyasova V, Fernandez SP, Laine J et al (2011) A genetically defined morphologically and functionally unique subset of 5-HT neurons in the mouse raphe nuclei. J Neurosci 31:2756-2768. doi:10. 1523/JNEUROSCI.4080-10.2011

Köhler CC-PV (1982) Somatostatin-like immunoreactive neurons in the hippocampus: an immunocytochemical study in the rat. Neurosci Lett 34:259-264

Liu C, Maejima T, Wyler SC et al (2010) Pet-1 is required across different stages of life to regulate serotonergic function. Nat Neurosci 13:1190-1198. doi:10.1038/nn.2623

Madisen L et al (1993) Regulation of fatty acid synthase gene expression: an approach for reducing fat accumulation. J Anim Sci 71:1957-1965. doi:10.1038/nn.2467

Mintz EM, Scott TJ (2006) Colocalization of serotonin and vesicular glutamate transporter 3-like immunoreactivity in the midbrain raphe of Syrian hamsters (Mesocricetus auratus). Neurosci Lett 394:97-100. doi:10.1016/j.neulet.2005.10.033

Mullen RJ, Buck CR, Smith AM (1992) NeuN, a neuronal specific nuclear protein in vertebrates. Development 116:201-211

Ohmura Y, Tanaka KF, Tsunematsu T et al (2014) Optogenetic activation of serotonergic neurons enhances anxiety-like behaviour in mice. Int $\mathbf{J}$ Neuropsychopharmacol. doi:10.1017/ S1461145714000637

Paxinos, G and Franklin KBJ (2012) Paxinos and Franklin's the mouse brain in stereotaxic coordinates, 4 th edn, Academic Press, ISBN:9780123910578

Pelosi B, Migliarini S, Pacini G et al (2014) Generation of Pet1210Cre transgenic mouse line reveals non-serotonergic expression domains of Pet1 both in CNS and periphery. PLoS One 9:e104318. doi:10.1371/journal.pone.0104318

Peters S, Slattery DA, Uschold-Schmidt N et al (2014) Dosedependent effects of chronic central infusion of oxytocin on anxiety, oxytocin receptor binding and stress-related parameters in mice. Psychoneuroendocrinology 42:225-236. doi:10.1016/j. psyneuen.2014.01.021

Rind HB, Russo AF, Whittemore SR (2000) Developmental regulation of tryptophan hydroxylase messenger RNA expression and enzyme activity in the raphe and its target fields. Neuroscience 101:665-677. doi:10.1016/S0306-4522(00)00402-4

Schmitz C, Hof PR (2005) Design-based stereology in neuroscience. Neuroscience 130:813-831. doi:10.1016/j.neuroscience.2004.08. 050 
Scott MM, Wylie CJ, Lerch JK et al (2005) A genetic approach to access serotonin neurons for in vivo and in vitro studies. Proc Natl Acad Sci USA 102:16472-16477. doi:10.1073/pnas. 0504510102

Serrats J, Artigas F, Mengod G, Cortés R (2003) GABA B receptor mRNA in the raphe nuclei: co-expression with serotonin transporter and glutamic acid decarboxylase. J Neurochem 84:743-752. doi:10.1046/j.0022-3042.2003.01557.x

Shutoh F, Ina A, Yoshida S et al (2008) Two distinct subtypes of serotonergic fibers classified by co-expression with vesicular glutamate transporter 3 in rat forebrain. Neurosci Lett 432:132-136. doi:10.1016/j.neulet.2007.12.050

Silva RCB, Gárgaro AC, Brandão ML (2004) Differential regulation of the expression of contextual freezing and fear-potentiated startle by 5-HT mechanisms of the median raphe nucleus. Behav Brain Res 151:93-101. doi:10.1016/j.bbr.2003.08.015

Somogyi J, Baude A, Omori Y et al (2004) GABAergic basket cells expressing cholecystokinin contain vesicular glutamate transporter type 3 (VGLUT3) in their synaptic terminals in hippocampus and isocortex of the rat. Eur $\mathrm{J}$ Neurosci 19:552-569. doi:10.1111/j.0953-816X.2003.03091.X

Spaethling JM, Piel D, Dueck H et al (2014) Serotonergic neuron regulation informed by in vivo single-cell transcriptomics. FASEB J 28:771-780. doi:10.1096/fj.13-240267

Stamp JA, Semba K (1995) Extent of colocalization of serotonin and GABA in the neurons of the rat raphe nuclei. Brain Res 677:39-49

Sterio D (1984) The unbiased estimation of number and sizes of arbitrary particles using the disector. J Microsc 134:127-136

Szőnyi A, Mayer MI, Cserép C et al (2014) The ascending median raphe projections are mainly glutamatergic in the mouse forebrain. Brain Struct Funct. doi:10.1007/s00429-014-0935-1
Varga V, Losonczy A, Zemelman BV et al (2009) Fast synaptic subcortical control of hippocampal circuits. Science 326:449-453. doi:10.1126/science.1178307

Vertes RP, Kocsis B (1997) Brainstem-diencephalo-septohippocampal systems controlling the theta rhythm of the hippocampus. Neuroscience 81:893-926

Vertes RP, Fortin WJ, Crane AM (1999) Projections of the median raphe nucleus in the rat. J Comp Neurol 407:555-582. doi:10. 1002/(SICI)1096-9861(19990517)407:4<555:AID-CNE7>3.3. $\mathrm{CO} ; 2-5$

Vong L, Ye C, Yang Z et al (2011) Leptin action on GABAergic neurons prevents obesity and reduces inhibitory tone to POMC neurons. Neuron 71:142-154. doi:10.1016/j.neuron.2011.05.028

Wang DV, Yau HJ, Broker CJ, Tsou JH, Bonci AIS (2015) Mesopontine median raphe regulates hippocampal ripple oscillation and memory consolidation. Nat Neurosci 18:728-735. doi: $10.1038 /$ nn.3998

West MJ, Slomianka LGH (1991) Unbiased stereological estimation of the total number of neurons in the subdivisions of the rat hippocampus using the optical fractionator. Anat Rec 231:482-497. doi:10.1002/ar.1092310411

Yizhar O, Fenno LE, Davidson TJ et al (2011) Optogenetics in neural systems. Neuron 71:9-34. doi:10.1016/j.neuron.2011.06.004

Zangrossi H, Graeff FG (2014) Serotonin in anxiety and panic: contributions of the elevated T-maze. Neurosci Biobehav Rev 46:397-406. doi:10.1016/j.neubiorev.2014.03.007 